\title{
Complex Aggregates in Relational Learning
}

\author{
Celine Vens \\ K.U.Leuven, Dept. of Computer Science \\ Celestijnenlaan 200A, 3001 Leuven, Belgium \\ E-mail: celine.vens@cs.kuleuven.be
}

In relational learning, one learns patterns from relational databases, which usually contain multiple tables that are interconnected via relations. Thus, an example for which a prediction is to be given may be related to a set of objects that are possibly relevant for that prediction. Relational classifiers differ with respect to how they handle these sets: some use properties of the set as a whole (using aggregation), some refer to properties of specific individuals, however, most classifiers do not combine both. This imposes an undesirable bias on these learners. This dissertation describes a learning approach that avoids this bias, using complex aggregates, i.e., aggregates that impose selection conditions on the set to aggregate on.

Keywords: relational learning, inductive logic programming, aggregation

\section{Introduction}

In relational learning, an example to be classified may be related to a set of other objects, via one-to-many or many-to-many relationships. Properties of this set, or of some of the objects it contains (or perhaps both) may be relevant for the classification. Among the many approaches to relational learning that currently exist, an important distinction can be made with respect to how they handle these relationships. Whereas inductive logic programming (ILP) [3] is biased towards testing the existence of specific elements in the set, other techniques use aggregate functions (such as $\max , \min , \operatorname{avg}, . .$.$) , which compute a feature of$ the set to summarize it. For example, in the context of a bank company dataset, an ILP system might predict a person to be positive (e.g., meaning he/she might be interested in buying a particular stock option) if that person has a savings account with balance higher than 20,000. Systems that use aggregates, on the other hand, could for example predict a person as positive if the sum of the balances on his accounts is higher than 20,000. We call this condition (the aggregate function together with the set to aggregate on and the comparison to a threshold) an aggregate condition.

Current relational learners usually do not combine both approaches, which imposes an undesirable bias on them [1]. Such a combination would involve aggregating over a subset of elements fulfilling specific conditions ("aggregating over a selection"). For example, a relevant criterion to determine whether a person is positive could be related to the sum (aggregation) of the balances on his savings (selection) accounts. Such combinations might be expected to naturally appear in certain patterns, but they are very difficult to construct for machine learning systems.

\section{Complex Aggregates: Combining Aggregates and Selections}

Complex aggregate conditions are aggregate conditions that impose a selection on the set to aggregate on. They can be used by learning systems to construct hypotheses. However, the use of complex aggregates presents several difficulties. First, the search space explored by a learning system is substantially increased. Second, the generality order of the hypotheses that is assumed by many relational learners is violated.

For example, ILP learners construct hypotheses (represented as first order clauses) by means of a refinement operator based on applying the following two basic operations: (1) applying a substitution to the current clause, or (2) adding a literal to the current clause. This refinement operator has the property that the refined clause is always more specific than the original clause, and hence introduces a generality order on the space of clauses, which allows efficient search strategies. Applying the same basic operations to the clause representing the set to aggregate on may yield a specialization or a generalization, or even none of both. 


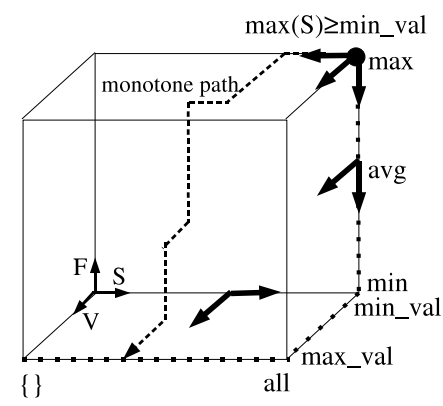

Fig. 1. Refinement cube for the class of generalized averages, defined as $\operatorname{avg}_{k}(S)=\left(\frac{\sum_{i}\left(x_{i}^{k}\right)}{n}\right)^{1 / k}$.

This behaviour is related to the monotonicity of aggregate conditions.

These issues imply that one either has to give up on efficiency or on completeness when searching the hypothesis space using classical refinement operators. In the thesis, we develop a refinement framework [5] that considers the complete search space, and traverses it in a general-to-specific, hence efficient, way. To this end we present several classes of aggregate functions, on which an ordering exists. Using this ordering, we propose a refinement operator that can refine an aggregate condition in three ways: (1) by changing the set to aggregate on, (2) by changing the threshold value and (3) by changing the aggregate function. This last dimension has never been explored before, but turns out to be crucial in order to obtain an efficient refinement strategy for aggregate conditions. The proposed refinement operator is visualized in refinement cubes (Fig. 1). Each point in the cube represents a complex aggregate condition, and can be reached, starting from one condition, in a finite number of refinements by following a monotone path, i.e., by making the previous condition more specific.

\section{Adding Complex Aggregates to First Order Decision Trees}

We include complex aggregates in the hypothesis language of TILDE [2], a first order decision tree learner. Complex aggregates can be introduced in the trees as refinement of a (simple) aggregate condition that occurs higher in the tree, or directly, by applying a lookahead technique. We argue that in this context, the generality ordering can not be violated, and classical refinement operators can be applied. To improve efficiency, we present two techniques: an application of the developed refinement framework and an upgrade to a first order random forest inducer [4].

We have also upgraded TILDE to a first order model tree learning system and investigated the use of complex aggregates in the linear models built in the leaves. The main contribution in this work is the development of an efficient heuristic function suitable for learning model trees. Other issues include how to deal with global effects and undefined aggregates.

\section{Conclusions}

We have combined aggregates and selections by introducing complex aggregates. We have proposed a refinement framework for constructing hypotheses with complex aggregates, and have implemented it in a first order decision tree learner. Other contributions are the development of a first order random forest inducer and a first order model tree inducer, both using complex aggregates.

The full dissertation is available at http: //www . cs.kuleuven. be/ celine/phd.

\section{Acknowledgements}

The dissertation was supervised by Hendrik Blockeel. Celine Vens is supported by the Research Fund K.U.Leuven.

\section{References}

[1] H. Blockeel and M. Bruynooghe. Aggregation versus selection bias, and relational neural networks. In IJCAI-2003 Workshop on Learning Statistical Models from Relational Data, SRL-2003, Acapulco, 2003.

[2] H. Blockeel and L. De Raedt. Top-down induction of first order logical decision trees. Artificial Intelligence, 101(1-2):285-297, June 1998.

[3] S. Muggleton, editor. Inductive Logic Programming. Academic Press, 1992.

[4] Anneleen Van Assche, Celine Vens, Hendrik Blockeel, and Sašo Džeroski. First order random forests: Learning relational classifiers with complex aggregates. $\mathrm{Ma}$ chine Learning, 64(1-3):149-182, 2006.

[5] Celine Vens, Jan Ramon, and Hendrik Blockeel. Refining aggregate conditions in relational learning. In Proc. of the 10th European Conf. on Principles of Data Mining and Knowledge Discovery. Springer, 2006. 